Pacific Journal of 


\title{
BEST CONSTANTS IN A CLASS OF INTEGRAL INEQUALITIES
}

\author{
DAVID W. BOYD
}

In this paper a method is developed for determining best constants in inequalities of the following form:

$$
\int_{a}^{b}|y|^{p}\left|y^{(n)}\right|{ }^{q} w(x) d x \leqq K\left\{\int_{a}^{b}\left|y^{(n)}\right| r m(x) d x\right\}^{(p+q) / r},
$$

where $y(a)=y^{\prime}(a)=\cdots=y^{(n-1)}(a)=0$ and $y^{(n-1)}$ is absolutely continuous.

It is first shown that for a certain class of $m$ and $w$, equality can be attained in the inequality. Applying variational techniques reduces the determination of the best constant to a nonlinear eigenvalue problem for an integral operator. If $m$ and $w$ are sufficiently smooth this reduces further to a boundary value problem for a differential equation. The method is illustrated by determining the best constants in case $(a, b)$ is a finite interval, $m(x) \equiv w(x) \equiv 1$, and $n=1$.

A number of special cases of the inequality have been studied but usually without obtaining best constants. An exception to this is the case $n=1, q=0, p=r$ which was studied very thoroughly by Beesack [1], who gave a direct method for determining best constants. The method of [1] was modified by Boyd and Wong [5] to apply to the case $n=1, q=1, r=p+1$. Recently Beesack and Das [2] obtained constants for the case $n=1, r=p+q$ but these were not in general best possible.

We shall state our result only for $n=1$ although it will be clear that the analogous result for $n>1$ is valid. In our closing remarks we indicate a number of other inequalities to which the method of this paper applies.

1. Preliminaries. Throughout we assume that $p, q, r, a, b$ are real numbers satisfying $p>0, r>1,0 \leqq q<r$ and $-\infty \leqq a<b \leqq \infty$. The functions $m$ and $w$ are measurable and positive almost everywhere. We write $d \mu(x)=m(x) d x$ and

$$
\|f\|_{s}=\left\{\int_{a}^{b}|f|^{s} d \mu\right\}^{1 / s} \text { for } 0<s<\infty .
$$

The space $L_{m}^{s}$ is the set of functions with $\|f\|_{s}<\infty$, with the usual identification. We shall use the notation $f_{n} \rightarrow f$ if $\left\|f_{n}-f\right\|_{s} \rightarrow 0$, and if $s \geqq 1$ so $L_{m}^{s}$ is a Banach space, we write $f_{n} \stackrel{w}{\longrightarrow} f$ for weak convergence in $L_{m}^{s}$. We denote the dual of $L_{m}^{s}$ by $L_{m}^{s^{\prime}}$ so for $s>1, s^{\prime}=s /(s-1)$. We shall consider integral operators of the type 


$$
T f(x)=\int_{a}^{b} k(x, t) f(t) d \mu(t),
$$

where $k(x, t) \geqq 0$ a.e. A function $f$ is in the domain of $T$ if

$$
T|f|(x)<\infty \quad \text { a.e. }
$$

For Theorem 1, the operator $T$ becomes

$$
T_{1} f(x)=w(x)^{1 / p} m(x)^{-1 / p} \int_{a}^{x} f(t) d t,
$$

so that $k(x, t)=w(x)^{1 / p} m(x)^{-1 / p} m(t)^{-1} \chi_{[a, x]}(t)$. A necessary and sufficient condition for the domain of $T_{\mathrm{t}}$ to contain $L_{m}^{r}$ is that

$$
\int_{a}^{x} m(t)^{-1(r-1)} d t<\infty \quad \text { for } \quad a \leqq x<b .
$$

This follows from Hölder's inequality and its converse.

If $T$ maps $L_{m}^{r} \rightarrow L_{m}^{s}$, where $s=p r /(r-q)$, with norm $\|T\|<\infty$, then we can define the functional $J$ on $L_{m}^{r}$ by

$$
J(f)=\int_{a}^{b}|T f|^{p}|f|^{q} d \mu .
$$

It then follows from Hölder's inequality that

$$
J(f) \leqq\|T\|^{p}\|f\|_{r}^{p+q} .
$$

\section{Main results.}

Theorem 1. Suppose that $w, m \in C^{1}(a, b)$, that $w(x)>0$ a.e. and $m(x)>0$ for $a<x<b$, that $p>0, r>1,0 \leqq q<r$, and that the operator $T_{1}$ defined by (2) is compact from $L_{m}^{r} \rightarrow L_{m}^{s}(s=p r /(r-q))$. Then the following eigenvalue problem $(P)$ has solutions $(y, \lambda)$ with $y \in C^{2}(a, b)$ and $y(x)>0, y^{\prime}(x)>0$ in $(a, b)$.

$$
(P)\left\{\begin{array}{l}
\text { ( i ) } \frac{d}{d x}\left(r \lambda y^{\prime r-1} m-q y^{p} y^{\prime q-1} w\right)+p y^{p-1} y^{\prime q} w=0 \\
\text { (ii) } \lim _{x \rightarrow a} y(x)=0 \text { and } \lim _{x \rightarrow b}\left(r \lambda y^{\prime r-1} m-q y^{p} y^{\prime q-1} w\right)=0 \\
\text { (iii) }\left\|y^{\prime}\right\|_{r}=1 .
\end{array}\right.
$$

There is a largest value $\lambda$ such that $(P)$ has a solution and if $\lambda^{*}$ denotes this value, then for any $f \in L_{m}^{r}$,

$$
\int_{a}^{b}\left|\int_{a}^{x} f\right|^{p}|f|^{q} w(x) d x \leqq \frac{r \lambda^{*}}{p+q}\left\{\int_{a}^{b}|f|^{r} m(x) d x\right\}^{(p+q) / r} .
$$

Equality holds in (5) if and only if $f=c y^{\prime}$ a.e. where $y$ is a solution 
of $(P)$ corresponding to $\lambda=\lambda^{*}$, and $c$ is any constant.

The proof will require two lemmas which we state in reasonable generality.

Lemma 1. Suppose that $p>0, r>1,0 \leqq q<r$, and that $T$, as defined by (1) is a compact operator from $L_{m}^{r} \rightarrow L_{m}^{s},(s=p r /(r-q))$. Let $J$ be defined by (3), and

$$
K^{*}=\sup \left\{J(f):\|f\|_{r} \leqq 1\right\} .
$$

Then, there is an element $f_{0} \in L_{m}^{r}$ with $\left\|f_{0}\right\|_{r}=1$ such that $J\left(f_{0}\right)=K^{*}$.

Proof. Since $J(f)<J(|f|)$ unless $f$ is of constant sign a.e., we can restrict consideration in (6) to $f \geqq 0$. Let $\left\{f_{n}\right\} \in L_{m}^{r}$ be a sequence with $f_{n} \geqq 0,\left\|f_{n}\right\| \leqq 1$ such that $J\left(f_{n}\right) \rightarrow K^{*}$. We begin by assuming $q>0$ so that $1<r / q<\infty$. By the weak sequential compactness of the unit balls of $L_{m}^{r}$ and $L_{m}^{r / q}([7]$, p. 68), and by the compactness of $T$, we may assume that there are functions

$$
f \in L_{m}^{r}, h \in L_{m}^{r / q}, g \in L_{m}^{s}
$$

such that $f_{n} \stackrel{w}{\longrightarrow} f, f_{n}^{q} \stackrel{w}{\longrightarrow} h, T f_{n} \rightarrow g$ in the appropriate spaces; clearly $T f=g$. Furthermore, by the uniform convexity of $L_{m}^{r}$ and $L_{m}^{r / q}$ we may assume that $f_{n}$ and $f_{n}^{q}$ are strongly $(C, 1)$-summable to their weak limits ([7], p. 462), so that

$$
\hat{f}_{n}=n^{-1} \sum_{k=1}^{n} f_{k} \longrightarrow f \text { and } \hat{h}_{n}=n^{-1} \sum_{k=1}^{n} f_{k}^{q} \longrightarrow h .
$$

Now, we have

$$
J\left(f_{n}\right)-\int_{a}^{b} g^{p} h d \mu=\int_{a}^{b}\left(\left(T f_{n}\right)^{p}-g^{p}\right) f_{n}^{q}+\int_{a}^{b} g^{p}\left(f_{n}^{q}-h\right) .
$$

Now, since $f_{n}^{q} \stackrel{w}{\longrightarrow} h$ in $L_{m}^{r / 4}$ and since $g^{p} \in L_{m}^{s / p}=L_{m}^{(r / q)^{\prime}}$, the second integral in the right member of (7) tends to zero as $n \rightarrow \infty$. To show that the first integral tends to zero we consider separately $0 \leqq p<1$ and $1 \leqq p<\infty$. If $0 \leqq p<1$, we use the inequality $\left|A^{p}-B^{p}\right| \leqq$ $|A-B|^{p}$ for $A \geqq 0, B \geqq 0$ to obtain

$$
\begin{aligned}
& \left|\int_{a}^{b}\left(T f_{n}\right)^{p} f_{n}^{q}-\int_{a}^{b} g^{p} f_{n}^{q}\right| \leqq \int_{a}^{b}\left|T f_{n}-g\right|^{p} f_{n}^{q} \\
& \leqq\left\|T f_{n}-g\right\|_{s}^{p}\left\|f_{n}\right\|_{r}^{q} \leqq\left\|T f_{n}-g\right\|_{s}^{p} .
\end{aligned}
$$

The second step follows from Hölder's inequality with exponents $s / p=$ $r /(r-q)$ and $r / q$. The final term in (8) tends to zero since $T f_{n} \rightarrow g$ in $L_{m}^{s}$.

In case $1 \leqq p<\infty$, we consider instead 
( 9 )

$$
\left|\left\{\int_{a}^{b}\left(T f_{n}\right)^{p} f_{n}^{q}\right\}^{1 / p}-\left\{\int_{a}^{b} g^{p} f_{n}^{q}\right\}^{1 / p}\right| \leqq\left\{\int_{a}^{b}\left|T f_{n}-g\right|^{p} f_{n}^{q}\right\}^{1 / p}
$$

by Minkowski's inequality. As in (8), the right member of (9) tends to zero. Thus, if $A_{n}=\int_{a}^{b}\left(T f_{n}\right)^{p} f_{n}^{q}$ and $B_{n}=\int_{a}^{b} g^{p} f_{n}^{q}$, we have that

$$
\left|A_{n}^{1 / p}-B_{n}^{1 / p}\right| \longrightarrow 0 \text {. }
$$

But $\left\{A_{n}\right\}$ and $\left\{B_{n}\right\}$ are bounded sequences $\left(A_{n}=J\left(f_{n}\right) \leqq\|T\|^{p}\right.$ by (4), $B_{n} \leqq\|g\|_{s}^{p} \leqq\|T\|^{p}$ by Hölder's inequality and $\left.T f_{n} \rightarrow g\right)$, and thus $\left|A_{n}-B_{n}\right| \leqq p\left|A_{n}^{1 / p}-B_{n}^{1 / p}\right| \cdot|| T \|^{p-1}$ shows that $A_{n}-B_{n} \rightarrow 0$ as required. Hence, we have

$$
K^{*}=\int_{a}^{b} g^{p} h d \mu .
$$

In case $q=0,(10)$ also holds with $h \equiv 1$, by a similar argument.

Now we show the existence of $f_{0}$ for which $J\left(f_{0}\right)=K^{*}$. The cases $0 \leqq q<1$ and $1 \leqq q<r$ are considered separately. If $0 \leqq q<1$, define $f_{0}=f$. Since $\varphi(t)=t^{q}$ is concave, we have

$$
\hat{f}_{n}^{q}=\left(n^{-1} \sum_{1}^{n} f_{k}\right)^{q} \geqq n^{-1} \sum_{1}^{n} f_{k}^{q}=\hat{h}_{n} .
$$

Now, since $\hat{f}_{n} \rightarrow f_{0}$ in $L_{m}^{r}$, we have

$$
\begin{aligned}
& \left|\int_{a}^{b} g^{p} f_{0}^{q}-\int_{a}^{b} g^{p} \hat{f}_{n}^{q}\right| \leqq \int_{a}^{b} g^{p}\left|f_{0}^{q}-\hat{f}_{n}^{q}\right| \\
& \quad \leqq \int_{a}^{b} g^{p}\left|f_{0}-\hat{f}_{n}\right|^{q} \leqq\|g\|_{s}^{p}\left\|f_{0}-\hat{f}_{n}\right\|_{r}^{q} \longrightarrow 0
\end{aligned}
$$

Similarly, $\int_{a}^{b} g^{p} \hat{h}_{n} \rightarrow \int_{a}^{b} g^{p} h$. Thus, combining (10), (11) and (12) we obtain

$$
\begin{aligned}
J\left(f_{0}\right) & =\int_{a}^{b} g^{p} f_{0}^{q}=\lim \int_{a}^{b} g^{p} \hat{f}_{n}^{q} \\
& \geqq \lim \int_{a}^{b} g^{p} \hat{h}_{n}=\int_{a}^{b} g^{p} h=K^{*} .
\end{aligned}
$$

However $\left\|f_{0}\right\|_{r} \leqq 1$ so $J\left(f_{0}\right) \leqq K^{*}$ and hence (13) implies $J\left(f_{0}\right)=K^{*}$ from which it is clear that $\left\|f_{0}\right\|_{r}=1$.

In case $1 \leqq q<r$, let $f_{0}=h^{1 / q}$. Now, instead of (11), we have $\hat{f}_{n}^{q} \leqq \hat{h}_{n}$. Since $\left\|\hat{h}_{n}-h\right\|_{r / q} \rightarrow 0$, and since $\left|\hat{h}_{n}^{1 / q}-h^{1 / q}\right| \leqq\left|\hat{h}_{n}-h\right|^{1 / q}$ we have

$$
\begin{aligned}
\left\|\hat{h}_{n}^{1 / q}-h^{1 / q}\right\|_{r} & =\left\{\int_{a}^{b} \mid \hat{h}_{n}^{1 / q}-h^{1 / q \mid r}\right\}^{1 / r} \\
& \leqq\left\{\int_{a}^{b}\left|\hat{h}_{n}-h\right|^{r / q}\right\}^{1 / r}=\left\|\hat{h}_{n}-h\right\|_{r / q}^{1 / q} .
\end{aligned}
$$


Thus $\hat{h}_{n}^{1 / q} \rightarrow h^{1 / q}=f_{0}$ in $L_{m}^{r}$ and since $T$ is continuous, $T \hat{h}_{n}^{1 / q} \rightarrow T f_{0}$ in $L_{m}^{r}$. However $\hat{f}_{n} \leqq \hat{h}_{n}^{1 / q}$ and $k(x, t) \geqq 0$ a.e. so $T \hat{f}_{n} \leqq T \hat{h}_{n}^{1 / q}$, a.e. and thus $T f=g \leqq T f_{0}$ a.e. Thus (10) implies $K^{*} \leqq J\left(f_{0}\right)$, which again means that $J\left(f_{0}\right)=K^{*}$ and $\left\|f_{0}\right\|_{r}=1$.

REMARK. A simple sufficient condition for $T$ to be compact from $L_{m}^{r} \rightarrow L_{m}^{s}$ is that $k$ have finite $\left(r^{\prime}, s\right)$-double norm. That is

$$
\text { || T } \mid \|=\left\{\int_{a}^{b}\left[\int_{a}^{b} k(x, t)^{r^{\prime}} d \mu(t)\right]^{s / r^{\prime}} d \mu(x)\right\}^{1 / s}<\infty
$$

(see [9], p. 319; the proof there applies even if $0<s<1$ ).

Using (4), we see that $K^{*} \leqq\|T\|^{p} \leqq\|T\|^{p}$ so (14) also supplies an upper bound for $K^{*}$ (rarely the best).

For the operator $T_{1}$ given by (2) one may calculate that

$$
\left\|T_{1}\right\|^{s}=\int_{a}^{b} w(x)^{r /(r-q)} m(x)^{-q /(r-q)}\left[\int_{a}^{x} m(t)^{-1 /(r-1)} d t\right]^{s / r^{\prime}} d x .
$$

In the paper of Beesack and Das [2], the following inequality is proved: If $p q>0, p+q>1, y(a)=0$ and $y$ is absolutely continuous, then

$$
\int_{a}^{b}|y|^{p}\left|y^{\prime}\right|{ }^{q} w(x) d x \leqq K_{1}(b, p, q) \int_{a}^{b}\left|y^{\prime}\right|^{p+q} m(x) d x,
$$

where $K_{1}(b, p, q)$ is explicitly given. The constant $K_{1}(b, p, q)$ equals the best constant $K^{*}$ if and only if for some $c \geqq 0$

$$
w(x)=c m(x)^{(q-1) /(r-1)}\left(\int_{a}^{x} m(t)^{-1 /(r-1)} d t\right)^{p(1-q) / q}(r=p+q) .
$$

The constant $K_{1}(b, p, q)$ given there is in fact equal to $(q / r)^{q / r}\left\|\left|T_{1} \|\right|^{p}\right.$, so, unless (17) holds we have

$$
K^{*}<K_{1}(b, p, q)<\left\|\mid T_{1}\right\|^{p} .
$$

Lemma 2. Suppose that $T$ is given by (1), and that $k(x, t)>0$ for almost all $(x, t)$ with $a \leqq t \leqq x \leqq b$. Let $p>0, r>1,0 \leqq q<r$, and suppose $T$ is a bounded operator from $L_{m}^{r} \rightarrow L_{m}^{s}$. Let $J$ be defined by (3), $K^{*}$ by (6). Let $f$ satisfy $\|f\|_{r}=1$ and $J(f)=K^{*}$. Then $f$ is of constant sign a.e. and

(a) $f \neq 0$ a.e.

(b) $f$ satisfies a.e. the equation

$$
r \lambda f^{r-1}(x)-q(T f)^{p}(x) f^{q-1}(x)-p \int_{a}^{b} k(t, x)(T f)^{p-1}(t) f^{q}(t) d \mu(t)=0,
$$

where $\lambda=\lambda^{*}=K^{*}(p+q) / r$. Furthermore $\lambda^{*}$ is the largest value of 
$\lambda$ for which (19) has a solution $f$ with $\|f\|_{r}=1$.

Proof. (a) We have seen that $f$ is of constant sign a.e. so we assume $f \geqq 0$ a.e. Let $E=\{x: f(x)=0\}$; we must show that $E$ is a null set. First choose a function $h \in L_{m}^{r}$ such that $h(x) \geqq 0$, and $h(x)>0$ if and only if $x \in E$. Such $h$ exist: if $\mu(E)<\infty$, take $h=$ $\chi_{E}$, while if $\mu(E)=\infty$, let

$$
E_{n}=E \cap[-n, n] \cap\{x: m(x) \leqq n\},
$$

so $\mu\left(E_{n}\right)<\infty$, and define $h=\sum \gamma_{n} \chi_{E_{n}}$ where $\left\{\gamma_{n}\right\}$ is chosen so $\gamma_{n}>0$ and $\sum \gamma_{n}^{r} \mu\left(E_{n}\right)<\infty$.

For $\varepsilon>0$, define $f_{\varepsilon}=f+\varepsilon h$, and let $F=T f, F_{\varepsilon}=T f_{\varepsilon}, H=T h$. since $J(f) /\|f\|_{r}^{p+q}$ is maximal, we have

$$
\begin{aligned}
0 & \leqq J\left(f_{\varepsilon}\right)-J(f) \leqq\left(\left\|f_{\varepsilon}\right\|_{r}^{p+q}-1\right) J(f) \\
& =\left\{\left(1+\varepsilon^{r}\|h\|_{r}^{r}\right)^{(p+q) / r}-1\right\} J(f) \\
& =\varepsilon^{r}\|h\|_{r}^{r} \gamma \xi^{r-1} J(f),\left(\text { where } \gamma=(p+q) / r, \text { and } 1<\xi<1+\varepsilon^{r}\|h\|_{r}^{r}\right. \\
& =0\left(\varepsilon^{r}\right) \text { as } \varepsilon \downarrow 0 .
\end{aligned}
$$

First assume that $q>0$, so if $C E=[a, b] \backslash E$, we may write

$$
J\left(f_{\varepsilon}\right)-J(f)=\varepsilon^{q} \int_{E} F_{\varepsilon}^{p} h^{q}+\int_{C E}\left(F_{\varepsilon}^{p}-F^{p}\right) f^{q} .
$$

From (20) and (21) we immediately deduce that

$$
0 \leqq \int_{E} F^{p} h^{q} \leqq \int_{E} F_{\varepsilon}^{p} h^{q}=0\left(\varepsilon^{r-q}\right) \rightarrow 0 \quad \text { as } \quad \varepsilon \downarrow 0 .
$$

Thus, $F(x)=0$ a.e. on $E$ so $k(x, t)=0$ a.e. on $E \times C E$.

Next, we note that $F(x)>0$ a.e. on $C E$, since $k(x, t)>0$ a.e. for $\mathrm{a} \leqq t \leqq x \leqq b$. Thus, for almost all $x$ in $C E$, we have $(d / d \varepsilon) F_{\varepsilon}^{p} f^{q}=$ $p F_{\varepsilon}^{p-1} H f^{q}$. Hence, if $0<\varepsilon<\varepsilon_{0}$ we have.

$$
\begin{aligned}
& p F^{p-1} H f^{q} \leqq \varepsilon^{-1}\left(F_{\varepsilon}^{p}-F^{p}\right) f^{q} \text { a.e. on } C E, p \geqq 1 \\
& p F_{\varepsilon_{0}}^{p-1} H f^{q} \leqq \varepsilon^{-1}\left(F_{\varepsilon}^{p}-F^{p}\right) f^{q} \text { a.e. on } C E, 0<p<1 \text {. }
\end{aligned}
$$

Thus, if $p \geqq 1,(20)$, (21) and (22) imply that

$$
0 \leqq \int_{C L} p F^{p-1} H f^{q} \leqq \varepsilon^{-1} \int_{C E}\left(F_{\varepsilon}^{p}-F^{p}\right) f^{q}=0\left(\varepsilon^{r-1}\right) \rightarrow 0 \quad \text { as } \quad \varepsilon \downarrow 0 .
$$

Thus, since $F(x) \neq 0$, we have $H(x)=0$ a.e. on $C E$. A similar argument using (20), (21) and (23) proves $H(x)=0$ a.e. on $C E$, if $0<p<1$. Thus $k(x, t)=0$ a.e. on $C E \times E$, and hence on $(E \times C E) \cup(C E \times E)$. But, since $k(x, t)>0$ a.e. for $a \leqq t \leqq x \leqq b$, the last sentence implies that $E x C E$ has plane measure zero and so either $\mu(E)=0$ or $\mu(C E)=0$. However, $\mu(C E)=0$ implies that $f=0$ a.e. contradicting $J(f)=K^{*} \neq 0$. 
Thus $\mu(E)=0$ as required.

In case $q=0$, (21) no longer holds. In this case, let

$$
A=\{x: F(x)=0\}
$$

so $k(x, t)=0$ a.e. on $A \times C E$. Clearly $\mu(A \cap C E)=0$, since $k(x, t)>0$ a.e. for $a \leqq t \leqq x \leqq b$. Instead of (21) we have

$$
J\left(f_{\varepsilon}\right)-J(f)=\varepsilon^{p} \int_{A} H^{p}+\int_{C A}\left(F_{\varepsilon}^{p}-F^{p}\right) .
$$

Proceeding as in (24), we use the second integral in (25) together with (20) to show that $H(x)=0$ a.e. on $C A$, so $k(x, t)=0$ a.e. on $C A \times E$. Now if $B=C A \cap E$ has $\mu(B)>0$, we would have $k(x, t)=0$ a.e. on $B \times B$ with contradicts $k(x, t)>0$ a.e. for $a \leqq t \leqq x \leqq b$ and thus $\mu(B)=\mu(E \backslash A)=0$. We already have shown that $\mu(A \backslash E)=0$. Thus $k(x, t)=0$ a.e. on $(A \times C E) \cup(C A \times E)$ means $k(x, t)=0$ a.e. on $(E \times C E) \cup$ $(C E \times E)$, which leads to a contradiction as before. (We note that if $p<r$, a simpler argument is available using the first integral in (25).)

(b) Consider the functional

$$
I(f)=\lambda\|f\|_{r}^{r}-J(f)=\int_{a}^{b}\left[\lambda f^{r}-(T f)^{p} f^{q}\right] d \mu .
$$

We shall show that if $J(f)=K^{*}$, and if $|h| \leqq f$, then for $\lambda=\lambda^{*}=$ $K^{*}(p+q) / r$, we have

$$
\delta I(f ; h)=\lim _{\varepsilon \rightarrow 0} \varepsilon^{-1}(I(f+\varepsilon h)-I(f))=0 .
$$

First, suppose that $|h| \leqq f$ and that $|\varepsilon| \leqq 1 / 2$. Now define $A(\varepsilon)=$ $J(f+\varepsilon h)$ and $B(\varepsilon)=\|f+\varepsilon h\|_{r}^{r}$. Then $A$ and $B$ are differentiable at $\varepsilon=0$, and

$$
\begin{aligned}
& A^{\prime}(0)=\int_{a}^{b}\left(p F^{p-1} f^{q} H+q F^{p} f^{q-1} h\right) d \mu \\
& B^{\prime}(0)=\int_{a}^{b} r f^{r-1} h d \mu .
\end{aligned}
$$

To see this, note that $(d / d \varepsilon) F_{\varepsilon}^{p} f_{\varepsilon}^{q}=p F_{\varepsilon}^{p-1} H f_{\varepsilon}^{q}+q F_{\varepsilon}^{p} f_{\varepsilon}^{q-1} h$ a.e. since $f>0$ a.e. by (a), and $F>0$ a.e. since $k(x, t)>0$ a.e. for $a \leqq t \leqq x \leqq b$, and thus $f_{\varepsilon}>0$ a.e., $F_{\varepsilon}>0$ a.e. for $|\varepsilon| \leqq \frac{1}{2}$ and $|h| \leqq f$.

But, we have

$$
\int_{a}^{b} F^{p-1} H f^{a} \leqq\left\{\int_{a}^{b} F^{s}\right\}^{(p-1) / s}\left\{\int_{a}^{b} H^{s}\right\}^{1 / s}\left\{\int_{a}^{b} f^{r}\right\}^{q / r}
$$

by Hölder's general inequality with exponents $s /(p-1), s$ and $r / q$. Similarly, one shows $F^{p} f^{q-1} h$ is integrable. And, for $|\varepsilon| \leqq \frac{1}{2}$ one may bound $\left|(d / d \varepsilon) F_{\varepsilon}^{p} f_{\varepsilon}^{q}\right|$ in terms of $F^{p-1} H f^{q}$ and $F^{p} f^{q-1} h$. For example, if $p \geqq 1, q \geqq 1$, one has 


$$
\left|\frac{d}{d \varepsilon} F_{\varepsilon}^{p} f_{\varepsilon}^{q}\right| \leqq\left(\frac{3}{2}\right)^{p+q-1}\left(p F^{p-1} H f^{q}+q F^{p} f^{q-1} h\right) \text { a.e. }
$$

with similar bounds if $0<p<1$ or $0 \leqq q<1$. Thus, Lebesgue's dominated convergence theorem gives (27). A similar argument gives (28).

By assumption $J(f) /\|f\|_{r}^{p+q}=A(0) / B(0)^{(p+q) / r}$ is maximal and hence

$$
\left.\frac{d}{d \varepsilon}\left(A(\varepsilon) B(\varepsilon)^{-(p+q) / r}\right)\right|_{s=0}=0 .
$$

Differentiating and using $A(0)=K^{*}$ and $B(0)=1$, we obtain

$$
A^{\prime}(0)-K^{*}((p+q) / r) B^{\prime}(0)=0
$$

or if we write $\lambda^{*}=K^{*}(p+q) / r$, we obtain

$$
\int_{a}^{b}\left(r \lambda^{*} f^{r-1} h-p F^{p-1} f^{q} H-q F^{p} f^{q-1} h\right) d \mu=0 .
$$

By Fubini's theorem we have

$$
\begin{aligned}
\int_{a}^{b} F^{p-1} f^{q} H & =\int_{a}^{b} F^{p-1}(x) f^{q}(x)\left(\int_{a}^{b} k(x, t) h(t) d \mu(t)\right) d \mu(x) \\
& =\int_{a}^{b} h(t)\left(\int_{a}^{b} k(x, t) F^{p-1}(x) f^{q}(x) d \mu(x)\right) d \mu(t) .
\end{aligned}
$$

Thus, if we write $T^{\prime}$ for the operator with kernel $k(t, x)$ we have from (31) and (32)

$$
\begin{aligned}
0 & =\int_{a}^{b} h(x)\left\{r \lambda^{*} f^{r-1}-q(T f)^{p} f^{q-1}-p T^{\prime}\left((T f)^{p-1} f^{q}\right)\right\} d \mu(x) \\
& =\int_{a} h(x) G(x) d \mu(x)
\end{aligned}
$$

To obtain (19) set $h(x)=f(x) \operatorname{sgn} G(x)$ in (33) and use the fact that $f(x) \neq 0$ a.e.

To see that $\lambda^{*}$ is the largest value of $\lambda$ for which a solution to (19) is possible with $\|f\|_{r}=1$, note that if (19) holds then (33) and hence (31) hold for any $|h| \leqq f$ with $\lambda$ in place of $\lambda^{*}$. Thus, setting $h=f$ in (31) (with $\lambda$ for $\lambda^{*}$ ), we obtain $r \lambda\|f\|_{r}^{r}-(p+q) J(f)=0$, and thus $\lambda=(p+q) J(f) / r \leqq(p+q) K^{*} / r=\lambda^{*}$.

REMARK. Part (a) of Lemma 2 may be strengthened by allowing $k$ to vanish on more extensive sets. However, the precise condition that is needed to insure $f \neq 0$ a.e. depends on the relationship of $p, q$ and $r$. For example, if $q>0$ and $p<r$, and if there are no sets $E$ with $\mu(E)>0$ and $\mu(C E)>0$ such that $k$ vanishes on $(E \times C E) \cup$ $(C E \times E)$ then for $f$ as in Lemma 2 , one has $f \neq 0$ a.e. 
Proof of Theorem 1. By Lemma 1, $\sup \left\{J(f):\|f\|_{r} \leqq 1\right\}=K^{*}<\infty$, and there is an $f \geqq 0$ with $\|f\|_{r}=1$ and $J(f)=K^{*}$. Since $m(x)>0$ and $w(x)>0$ a.e., Lemma 2 applies and we have $f \neq 0$ a.e. in $[a, b]$, and $f$ satisfies

$$
\lambda r f^{r-1}(x) m(x)-q F(x)^{p} f(x)^{q-1} w(x)-p \int_{x}^{b} F^{p-1} f^{q} w=0 \text { a.e. }
$$

where $F(x)=\int_{a}^{x} f(t) d t$.

We claim that by modifying $f$ on a set of measure zero, we will have $f \in C^{1}(a, b), f(x) \neq 0$ in $(a, b)$ and $f$ will satisfy (34) everywhere. To see this, rewrite (34) as

$$
f^{r-1}-A(x) f^{q-1}=B(x) \text { a.e. }
$$

where $A(x) \geqq 0$, and $B(x)>0$ for all $x \in(a, b)$.

Consider the equation $\zeta^{r-1}-\xi \zeta^{q-1}=\eta$. For $\eta>0, \xi \geqq 0$ this has a unique positive solution $\zeta=\varphi(\xi, \eta)$ which can be extended to be $C^{\infty}$ on an open region containing the set $\{(\xi, \eta): \xi \geqq 0, \eta \geqq 0, \xi+\eta>0\}$. To see this, consider the function $\psi(\zeta)=\zeta^{r-1}-\xi \zeta^{q-1}$ for fixed $\xi, r$ and $q$. First suppose $q \geqq 1$, and $\xi>0$, then $\psi^{\prime}(\zeta)$ has a single positive zero $\zeta_{0}=\zeta_{0}(\xi)$, and $\psi$ decreases from $\psi(0)=0$ to $\psi\left(\zeta_{0}\right)<0$ and is strictly increasing on $\left[\zeta_{0}, \infty\right)$ to $+\infty$. Thus $\psi(\zeta)=\eta$ has a unique solution for $\eta>0$ which we denote $\varphi(\xi, \eta)$. We define $\varphi(\xi, \eta)$ for $\xi>0$ and $0 \geqq \eta>\psi\left(\zeta_{0}(\xi)\right)$ to be that solution of $\eta=\psi(\zeta)$ with $\zeta>\zeta_{0}$. If $q \geqq 1$ and $\xi \leqq 0$, then $\psi$ is strictly increasing from $\psi(0)=0$, hence $\psi(\zeta)=\eta$ has a unique solution for $\eta \geqq 0$. Thus, for $q \geqq 1, \psi(\xi, \eta)$ is defined on an open set containing $Q=\{(\xi, \eta): \xi \geqq 0, \eta \geqq 0, \zeta+\eta>0\}$, and since $\psi^{\prime}(\varphi(\xi, \eta))>0$, the implicit function theorem shows that $\varphi \in C^{\infty}$. To show that $\varphi(\xi, \eta) \rightarrow \varphi(0,0)=0$ as $(\xi, \eta) \rightarrow(0,0)$ in $Q$, we note that if $0 \leqq \xi \leqq \delta, 0 \leqq \eta \leqq \delta$ and $\zeta_{1}=\alpha \delta^{1 /(r-1)}$ with $\alpha=2^{1 /(r-q)}$, then

$$
\psi\left(\zeta_{1}\right) \geqq \alpha^{r-1} \delta-\delta \alpha^{q-1} \delta^{(q-1) /(r-1)} \geqq \alpha^{q-1}\left(\alpha^{r-q}-1\right) \delta \geqq \delta \text { (if } \delta \leqq 1 \text { ). }
$$

Thus $\varphi(\xi, \eta) \leqq \alpha \delta^{1 /(r-1)}$ for $0 \leqq \xi \leqq \delta, 0 \leqq \eta \leqq \delta$ proving the assertion.

If $0 \leqq q<1$ and $\xi>0$, then $\psi$ is strictly increasing from $-\infty$ to $\infty$ on $(0, \infty)$ so $\psi(\zeta)=\eta$ has a unique solution for all $\eta$. If $0 \leqq q<1$ and $\xi<0$, then $\psi(\zeta) \rightarrow \infty$ as $\zeta \rightarrow 0+$ or $\zeta \rightarrow \infty$, and $\psi$ has a minimum at a point $\zeta_{0}$ where $\psi\left(\zeta_{0}\right)=\gamma|\xi|^{(r-1) /(r-q)}$ and $\gamma>0$. If $\xi=0, \psi(\xi)=\eta$ has a unique solution for $\eta \geqq 0$. Again we have $\varphi \in C^{\infty}$ on an open set containing $Q$ and that $\varphi(\xi, \eta) \rightarrow 0$ as $(\xi, \eta) \rightarrow(0,0)$ in $Q$.

Now, from (35), by modifying $f$ on a null set, we have

$$
f(x)=\varphi(A(x), B(x)) \text { for all } x \in(a, b) \text {. }
$$

If $w, m \in C^{1}$ then $A, B$ are absolutely continuous so (36) shows that $f$ 
is absolutely continuous. But then $F \in C^{1}$ so in fact, $A, B \in C^{1}$ and (36) shows that $f \in C^{1}$. That $f(x) \neq 0$ for $x \in(a, b)$ follows immediately from (36).

Now, defining $y=F$ and differentiating (34) once gives $(P)$ (i). The conditions (ii) and (iii) are apparent from (34). The problem $(P)$ thus has solutions for $\lambda=K^{*}(p+q) / r$. To identify the largest eigenvalue of $(P)$ as $K^{*}(p+q) / r$, we note that a solution of $(P)$ gives a solution of (34) and by Lemma 2 the largest eigenvalue of (34) is $K^{*}(p+q) / r$.

The inequality (5) and the statement concerning equality are now obvious.

REMARK. If $m(x)>0$ and $w(x)>0$ for all $x \in[a, b]$, and if $q>0$, then $A(x)>0$ unless $x=a$ and $B(x)>0$ unless $x=b$. Hence equation (36) shows that $f(x)>0$ for all $x \in[a, b]$; and $f \in C^{1}[a, b]$. We also note that if $\lim _{x \rightarrow b} A(x)$ is finite and $\lim _{x \rightarrow a} B(x)$ is finite then $f(a)<\infty$ and $f(b)<\infty$. This will be used in $\S 3$.

3. Some inequalities on a finite interval. As an application of Theorem 1, we obtain the best constants in case $(a, b)$ is a finite interval and $m(x) \equiv w(x) \equiv 1$. We immediately consider

$$
\int_{0}^{1}|y|^{p}\left|y^{\prime}\right|^{q} d x \leqq K(p, q, r)\left\{\int_{0}^{1}\left|y^{\prime}\right|^{r} d x\right\}^{(p+q) / r}
$$

where $y$ is absolutely continuous and $y(0)=0$.

Some special cases of (37) are known. The case $q=0, p=r=2 k$ ( $k$ a positive integer) is inequality 256 of [8], which was derived there by classical variational methods using the Weierstrass sufficient condition. This case was handled by elementary methods in [3]. Opial's inequality is the case $p=q=1, r=2$. If $q=1, r=p+1$, the best constant can be obtained by Hölder's inequality (see [5], for example). The case $r=p+q$ was considered in [6] but the best constant was found only when $q=1$ or $r=1$.

Note that if $q>r$, there is no inequality of the form (37), since for $y(x)=1-(1-x)^{1-r}, q^{-1}<\gamma<r^{-1}$, the left member of (37) is infinite while $\left\|y^{\prime}\right\|_{r}<\infty$. The case $p=0$ is simply Hölder's inequality with $K(0, q, r)=1$.

THEOREM 2. For $r \geqq 1, p>0,0 \leqq q \leqq r$, the inequality (37) is valid with a finite constant $K(p, q, r)$. The best such constant is given by the following expressions

(a) if $p>0, r>1,0 \leqq q<r$, then

$$
K(p, q, r)=\frac{(r-q) p^{p}}{(r-1)(p+q)} \beta^{p+q-r} I(p, q, r)^{-p}
$$


where

$$
\beta=\left\{\frac{p(r-1)+(r-q)}{(r-1)(p+q)}\right\}^{1 / r}
$$

and

$$
I(p, q, r)=\int_{0}^{1}\left\{1+\frac{r(q-1)}{r-q} t\right\}^{-\langle q+p+r p) / r p}\{1+(q-1) t\} t^{1 / p-1} d t .
$$

(b) If $r=1$, then

$$
K(p, q, 1)=\left\{\begin{array}{ll}
q^{q}(p+q)^{-q}, & q>0 \\
1 & , q=0
\end{array} .\right.
$$

( c) If $q=r$, then

$$
K(p, r, r)=\frac{r p^{p}}{p+r}\left(\frac{p}{p+r}\right)^{p / r} B\left(\frac{1}{r}+1, \frac{1}{p}\right)^{-p} .
$$

If $r=1, q=0$, there is strict inequality for all $y \neq \equiv 0$ while in all other cases there is equality only for multiples of a single function $y(p, q, r, x)$ which is in $C^{\infty}(0,1)$, and is concave if $0 \leqq q<1$, convex if $q>1$, linear if $q=1$.

For special cases of (a), (28) reduces to a simpler form. First, if $r=p+q$, we have

$$
K(p, q, p+q)=q(p+q)^{p-1}\{p L(p, q)+q\}^{-p}, q \neq 0
$$

where

$$
L(p, q)=\int_{0}^{1} \frac{d s}{1-k s^{p}}, k=\frac{(p+q)(q-1)}{(p+q-1) q} .
$$

In particular,

$$
K(1, q, 1+q)=\left\{1+\frac{2 q}{q^{2}-1} \log q\right\}^{-1}, q \neq 0,1 .
$$

If $q=0$, and $r>1$, we have

$$
\begin{aligned}
K(p, 0, r) & =A(p, r)^{p} \\
A(p, r) & =\left(r^{\prime}\right)^{1 / p} p^{1 / r^{\prime}}\left(p+r^{\prime}\right)^{1 / r-1 / p} B\left(1 / p, 1 / r^{\prime}\right)^{-1},
\end{aligned}
$$

where $r^{\prime}=r /(r-1)$. Note that $A(p, r)$ is the norm of the mapping $T: L^{r} \rightarrow L^{p}$ where here $T f(x)=\int_{0}^{x} f(t) d t$. By (4), if $\|f\|_{r}=1$ we have $J(f) \leqq\|T\|^{p}$, where $\|T\|$ is the norm of $T$ as a mapping from $L_{m}^{r} \rightarrow$ $L_{m}^{s},(s=p r /(r-q))$, and so we always have $K(p, q, r) \leqq A(s, r)^{p}$. 
We also note that in (38), if $q \neq 1$, one may make the replacement (41)

$$
(r-q) I(p, q, r)^{-p}=r^{p+1}(q-1) I_{1}(p, q, r)^{-p},
$$

where

$$
I_{1}(p, q, r)= \pm(r-q) \int_{0}^{ \pm T} t^{1 / p}(1 \mp t)^{\gamma-1} d t+r \int_{0}^{ \pm T} t^{(1 / p)-1}(1 \mp t)^{\gamma} d t,
$$

where $T_{1}=1-[(r-q) / q(r-1)], \gamma=(p+q-r) / r p$, and the upper sign is used with $q>1$, the lower sign with $q<1$.

Proof. In case (a), Theorem 1 applies since certainly $\left\|T_{1}\right\| \mid<\infty$. We seek solutions of the problem $(P)$. We first observe that by the remark at the end of $\S 2$, we have $y \in C^{2}[a, b]$ and $0<y^{\prime}(0)<\infty, 0<$ $y^{\prime}(1)<\infty$ except in case $q=0$ when we have $y^{\prime}(1)=0$. To see this note that the functions $A$ and $B$ which appear in (35) are here just

$$
A(x)=q(\lambda r)^{-1} y(x)^{p}, B(x)=p(\lambda r)^{-1} \int_{x}^{1} y(t)^{p-1} y^{\prime}(t)^{q} d t .
$$

But $y(1)=\int_{0}^{1} y^{\prime}(t) d t \leqq\left\|y^{\prime}\right\|_{r}<\infty$, so $A(1)<\infty$, and

$$
\int_{0}^{1} y^{p-1} y^{\prime q} \leqq\left\{\int_{0}^{1} y^{r(p-1) /(r-q)}\right\}^{(r-q) / r}\left\|y^{\prime}\right\|_{r}^{q}
$$

which shows that $B(0)<\infty$.

Notice that equation (i) of $(P)$ has the integrating factor $y^{\prime}$ from which we obtain

$$
(r-1) \lambda y^{\prime r}-(q-1) y^{p} y^{q}=\alpha \lambda,
$$

where $\alpha$ is a constant which is evaluated by using $\left\|y^{\prime}\right\|_{r}=1$ and $J\left(y^{\prime}\right)=r \lambda /(p+q)$. Thus we have

$$
\alpha=(r-1)-\frac{(q-1) r}{p+q}=\frac{p r-p-q+r}{p+q}>0 .
$$

Solving (42) for $y=\lambda^{1 / p} G\left(y^{\prime}\right)$ and differentiating leads to a variables separable equation for $y^{\prime}$, and if we write $z=y^{\prime}$ we have, for $q \neq 1$

$$
\begin{aligned}
d x & =\frac{\lambda^{1 / p}}{p}\left\{\frac{(r-1) z^{r-q}-\alpha z^{-q}}{q-1}\right\}^{(1 / p)-1} \\
& \times\left\{\frac{r-1)(r-q) z^{r-q-2}+\alpha q z^{-q-2}}{q-1}\right\} d z .
\end{aligned}
$$

To obtain boundary conditions, we use (42) and (ii) and thus, since $z(0) \neq 0$ and $z(1) \neq 0$ for $q \neq 0$, we obtain 


$$
z(0)^{r}=\alpha /(r-1) \text { and } z(1)^{r}=\alpha q /(r-q) .
$$

We now integrate (44) from $x=0$ to $x=1$ using (45) and make the change of variables (for $q \neq 1$ )

$$
t=\frac{r-q}{r \alpha} \cdot \frac{(r-1) z^{r}-\alpha}{q-1}
$$

which leads to equation (38).

For $q=1$, we note that $I(p, 1, r)=p, \beta=1$, and so (38) gives $K(p, 1, r)=(p+1)^{-1}$ which is the correct result by our earlier remarks. In the equation $y=\lambda^{1 / p} G\left(y^{\prime}\right), G$ is increasing if $q>1$ and decreasing if $q<1$. Thus, since $y$ is increasing, we must have $y^{\prime}$ increasing if $q>1$ and decreasing if $q<1$. The solution to problem $(P)$ with $\lambda=\lambda^{*}$ can be seen to be unique in the following way. We know that a solution of $(P)$ must satisfy $y=\lambda^{1 / p} G\left(y^{\prime}\right)$ and thus also $y^{\prime}=$ $\lambda^{1 / p} G^{\prime}\left(y^{\prime}\right) y^{\prime \prime}$, and hence $y^{\prime}$ satisfies

$$
\lambda^{1 / p} \int_{y^{\prime}(0)}^{y^{\prime}(x)} G^{\prime}(z) \frac{d z}{z}=x
$$

But, for $q \neq 1, G^{\prime}(z)$ does not change sign on the interval from $y^{\prime}(0)$ to $y^{\prime}(1)$ so $(46)$ has a unique solution for $y^{\prime}(x)$, and hence $(P)$ has a unique solution when $\lambda=\lambda^{*}$.

To obtain the alternate expression for $(r-q) I(p, q, r)^{-p}$ given in (41), we make the change of variable $t=1-\alpha(r-1)^{-1} z^{-r}$ in (44).

To obtain the formula (40), we make the following change of variables in (38)

$$
1+\frac{r(q-1)}{r-q} t=\left(1-\frac{r(q-1)}{q(r-1)} s^{p}\right)^{-1}=R(s)^{-1} .
$$

Then $t=(p / q(r-1)) s^{p} R(s)^{-1}$, and $t=0,1$ correspond to $s=0,1$ and one has

$$
\begin{aligned}
I(p, q, r) & =\operatorname{const}\left\{\int_{0}^{1} R(s)^{(q / r p)+(1 / r)+1-(2 / p)-1}\left(p R(s)^{-1}+q\right) d s\right\} \\
& =\text { const }\{p L(p, q)+q\}, \text { since } r=p+q .
\end{aligned}
$$

The formula (40) can be obtained in a more direct way by making the substitution $u=(q / r \lambda)^{1 / p}\left(y / y^{\prime}\right)$ in equation (i), where we assume $q \neq 0$ so $y^{\prime}(x)>0$ for $x \in[a, b]$. Then the conditions (ii) give $u(0)=0$, $u(1)=1$, and equation (i) reduces to

$$
(r-1)\left\{1-\frac{r(q-1)}{q(r-1)} u^{p}\right\}=p\left(\frac{r \lambda}{q}\right)^{1 / p} u^{\prime} \quad(r=p+q) .
$$

Separating variables and integrating gives (40). 
For case (b), let $z(x)=\int_{0}^{x}\left|y^{\prime}\right| d t$, and then if $q>0$

$$
\begin{aligned}
\int_{0}^{1}|y|^{p}\left|y^{\prime}\right|^{q} d x & \leqq \int_{0}^{1}\left(z^{p / q} z^{\prime}\right)^{q} d x \\
& \leqq\left\{\int_{0}^{1} d x\right\}^{1 / q}\left\{\int_{0}^{1} z^{p / q} z^{\prime} d x\right\}^{q}=\left(\frac{q}{p+q}\right)^{q} z^{p+q},
\end{aligned}
$$

using Hölder's inequality with exponents $1 / q>1$ and $1 /(1-q)$. Equality holds only if $z^{p / q} z^{\prime}$ is constant, and $y=z$ which means $y(x)=c x^{q /(p+q)}$. For $q=0$, we have

$$
\int_{0}^{1}|y|^{p} d x \leqq \int_{0}^{1}|z|^{p} d x \leqq z(1)^{p}=\int_{0}^{1}\left|z^{\prime}(x)\right| d x .
$$

Equality holds only if $y=z$, and $z(x)=z(1)$ for all $x$, so $y(x)=z(x) \equiv 0$.

For case (c), we let $q \rightarrow r-$ in formula (38), using the equation (41) to evaluate $\lim (r-q) I(p, q, r)^{-p}$. This shows that the best constant is given by (39), because if $y^{\prime} \in L^{r}$ and $q<r$, then

$$
\int_{0}^{1}|y|^{p}\left|y^{\prime}\right|^{q} \rightarrow \int_{0}^{1}|y|^{p}\left|y^{\prime}\right|^{r}
$$

by dominated convergence. To handle the case of equality we cannot apply Lemma 2 directly since the proof of Lemma 2(a) used $r>q$. However, if there is an $f$ with $J(f)=K(p, r, r)=K^{*}$ then we know that $f \geqq 0$ a.e. Now referring to the proof of Lemma 2(a), since $r>1$ we do have (24) which proves that if $E=\{x: f(x)=0\}$, then $k(x, t)=0$ a.e. on $C E \times E$. This means that

$$
(C E \times E) \cap\{(x, t): 0 \leqq t \leqq x \leqq 1\}
$$

is a set of measure zero. This implies that $E$ differs from an interval $[c, 1]$ by a set of measure zero. To see this, let

$$
c=\sup \{x \leqq 1:[0, x] \cap E \text { is of measure zero }\},
$$

and let $d=\inf \{x \geqq 0:[x, 1] \cap C E$ is of measure zero $\}$. Clearly $d \geqq c$. But, if $d>c$, and $e=(d+c) / 2$, then $[c, e] \cap E$ and $[e, d] \cap C E$ have positive measure; but then $C E \times E$ intersects $\{(x, t): 0 \leqq t \leqq x \leqq 1\}$ in a set of positive measure which is absurd.

However if equality held for such an $f$, we would have (writing $\left.f(x)=y^{\prime}(x)\right)$,

$$
\int_{0}^{c}|y|^{p}\left|y^{\prime}\right|^{r} d x=K^{*}\left\{\int_{0}^{c}\left|y^{\prime}\right|^{r} d x\right\}^{(p+r) / r} .
$$

Define $z(t)=y(c t)$ so $z^{\prime}(t)=c y^{\prime}(c t)$, and from (49) we obtain 


$$
\int_{0}^{1}|z|^{p}\left|z^{\prime}\right|^{r} d x=K^{*} c^{-p(r-1) / r}\left\{\int_{0}^{1}\left|z^{\prime}\right|^{r} d x\right\}^{(p+r) / r} .
$$

But, if $c<1$, then $K^{*} c^{-p(r-1) / r}>K^{*}$ contradicting the maximality of $K^{*}$. Thus $c=1$, so $f(x)=y^{\prime}(x)>0$ a.e. on $[0,1]$.

Still proceeding on the assumption that there exists an $f$ with $J(f)=K^{*}$ we have shown $f(x)>0$ a.e. on $[0,1]$, so the proof of Lemma $2(\mathrm{~b})$ is valid and $f$ satisfies

$$
\lambda r f^{r-1}(x)-r F(x)^{p} f^{r-1}(x)-p \int_{x}^{1} F^{p-1} f^{r} d t=0 \text { a.e. }
$$

where $F(x)=y(x)=\int_{0}^{x} f(t) d t$, and $\lambda=(p+q) K^{*} / r$.

If $x$ is any point where $f(x)>0$ and (51) holds then (51) shows that $\lambda f^{r-1}(x)>F^{p}(x) f^{r-1}(x)$, so $F^{p}(x)<\lambda$ a.e. But $F$ is strictly increasing so $F(x)^{p}<\lambda$ for $0 \leqq x<1$. Now we can solve (51) for $f$ and obtain

$$
f(x)=\varphi(A(x), B(x)) \text { for almost all } x \in[0,1) \text {. }
$$

where $\varphi(\xi, \eta)=(\eta /(1-\xi))^{1 /(r-1)}, A(x)=\lambda^{-1} F(x)^{p}<1$ for $0 \leqq x<1$, and $B(x)=p(r \lambda)^{-1} \int_{x}^{1} F^{p-1} f^{r} d t>0$ for $0 \leqq x \leqq 1$. Now we proceed as in the proof of Theorem 1. If we modify $f$ on a null set so that it satisfies (52) everywhere then we obtain $f \in C^{1}[0,1)$, and $f(x)>0$ for $0 \leqq x<1$. Thus we see that if $J(f)=K^{*}$ and $y^{\prime}=f$ then $y$ must be a solution of the problem $(P)$, with $\lambda=\lambda^{*}=(p+r) K^{*} / r$. But a solution of $(P)$ must be a solution of (42) (with $q=r$ ) which is

$$
\lambda y^{\prime r}-y^{p} y^{\prime r}=p \lambda /(p+r) .
$$

However if (53) has a solution then it must also satisfy

$$
\int_{0}^{y(x)}\left(\lambda-u^{p}\right)^{1 / r} d u=(p \lambda /(p+r))^{1 / r} x .
$$

To see that (54) has a unique solution for $0 \leqq x \leqq 1$, we note that

$$
\int_{0}^{\lambda^{1 / p}}\left(\lambda-u^{p}\right)^{1 / r} d u=p^{-1} \lambda^{1 / p} B\left(\frac{1}{r}+1, \frac{1}{p}\right)=(p \lambda /(p+r))^{1 / r},
$$

using the formula for $K(p, r, r)=K^{*}$ and $\lambda=(p+r) K^{*} / r$. Since $\lambda-u^{p}>0$ for $0 \leqq u<\lambda^{1 / p}$, (54) has a unique solution $y=y(x)$ which is strictly increasing and has $y(0)=0, y(1)=\lambda^{1 / p}$. To complete the proof we must show that $y$ in fact satisfies (i), (ii) and (iii) of $(P)$. By the implicit function theorem $y \in C^{2}(a, b)$, and differentiating (54) twice shows that $y$ satisfies (i). Clearly $y(0)=0$. For the other part of (ii), we note that for $0 \leqq x<1$, we have

$$
\left(\lambda-y^{p}(x)\right)^{1 / r} y^{\prime}(x)=(p \lambda /(p+r))^{1 / r},
$$


and since $y^{p}(x) \rightarrow \lambda$ as $x \rightarrow 1-$, we have $y^{\prime}(x) \rightarrow \infty$ as $x \rightarrow 1-$. But this means that

$$
\left(\lambda-y^{p}(x)\right) y^{\prime}(x)^{r-1}=(p \lambda /(p+r)) y^{\prime}(x)^{-1} \longrightarrow 0 \text { as } x \longrightarrow 1
$$

To verify that $\left\|y^{\prime}\right\|_{r}=1$, let us first introduce the function $g$ by

$$
g(t)=(p \lambda)^{-1 / r}(p+r)^{1 / r} \int_{0}^{t}\left(\lambda-u^{p}\right)^{1 / r} d u,
$$

so $g(y(x))=x$ for $x \in[0,1]$ and hence $y(g(t))=t$ for $t \in\left[0, \lambda^{1 / p}\right]$. Now

$$
\begin{aligned}
\int_{0}^{1} y^{\prime}(x)^{r} d x & =\frac{p \lambda}{p+r} \int_{0}^{1} \frac{d x}{\lambda-y^{p}(x)} \\
& =\left(\frac{p \lambda}{p+r}\right)^{1-(1 / r)} \int_{0}^{\lambda^{1 / p}}\left(\lambda-t^{p}\right)^{(1 / r)-1} d t,
\end{aligned}
$$

where we use the change of variable $x=g(t)$. Now using the formula for $\lambda$, we obtain

$$
\int_{0}^{1} y^{\prime}(x)^{r} d x=\frac{p}{p+r} B\left(\frac{1}{r}, \frac{1}{p}\right) / B\left(\frac{1}{r}+1, \frac{1}{p}\right)=1 .
$$

REMARKs. (1) As was mentioned above, the method of this paper applies to inequalities of the form (1) with $n>1$. In this case $T$ becomes

$$
w(x)^{1 / p} m(x)^{-1 / p} \int_{a}^{x} \frac{(x-t)^{n-1}}{(n-1) !} f(t) d t .
$$

A discussion of the special case $p=q=1, r=2$ will be found in [4] where, for $m(x) \equiv w(x) \equiv 1,[a, b]=[0,1]$, the best constant is shown to be asymptotic to $1 / 4 n$ !

(2) The method is equally applicable to inequalities in which the function $y$ is restricted by other boundary conditions. For example, if $[a, b]$ is a finite interval we may treat

$$
\begin{aligned}
\int_{a}^{b} y^{p} y^{\prime \prime q} w(x) d x & \leqq K\left\{\int_{a}^{b} y^{\prime \prime r} m(x) d x\right\}^{(p+q) / r} \\
y(a) & =y(b)=0
\end{aligned}
$$

In this case, if $f$ is a given function in $L_{m}^{r}$, the boundary value problem $y^{\prime \prime}=-f, y(a)=y(b)=0$ has a solution $y(x)=\int_{a}^{b} G(x, t) f(t) d t$, where $G(x, t) \geqq 0$ a.e. Hence our lemmas apply.

(3) When Theorem 1 is specialized to the situation studied by Beesack in [1] $(q=0, p=r)$, the results are not as general as his. This is because we can effectively handle only those inequalities where 
we can insure in advance that equality is possible. There is some compensation in the fact that the existence of solutions to the EulerLagrange equations $(P)$ is a conclusion of our theorem rather than a hypothesis as in [1] and [5].

\section{REFERENCES}

1. P. R. Beesack, Hardy's inequality and its extensions, Pacific J. Math. 11 (1961), 39-61.

2. P. R. Beesack, and K. M. Das, Extensions of Opial's inequality, Pacific J. Math. 26 (1968), 215-232.

3. Donald C. Benson, Inequalities involving integrals of functions and their derivatives, J. Math. Anal. Appl. 17 (1967), 292-308.

4. D. W. Boyd, Best constants in inequalities related to Opial's inequality, J. Math. Anal. Appl. 25 (1969), 378-387.

5. D. W. Boyd, and J. S. W. Wong, An extension of Opial's inequality, J. Math. Anal. Appl. 19 (1967), 100-102.

6. K. M. Das, An inequality similar to Opial's inequality, Proc. Amer. Math. Soc. (to appear)

7. N. Dunford, and J. T. Schwartz, Linear operators, Part I, Interscience, 1957.

8. G. H. Hardy, J. E. Littlewood and G. Polya, Inequalities, Cambridge, 1934.

9. A. C. Zaanen, Linear Analysis, North Holland-Amsterdam, 1953.

Received September 27, 1968. This work was supported in part by National Science Foundation grant GP-6111.

CALifornia INSTItUTe of TECHNOLOGY

Pasadena, California 



\title{
PACIFIC JOURNAL OF MATHEMATICS
}

\author{
EDITORS
}

H. ROYDEN

Stanford University

Stanford, California

R. R. PhelPS

University of Washington

Seattle, Washington 98105
J. DUGUNDJI

Department of Mathematics

University of Southern California

Los Angeles, California 90007

RICHARD ARENS

University of California

Los Angeles, California 90024

\section{ASSOCIATE EDITORS}
E. F. BECKENBACH
B. H. NeumanN
F. WOLF
K. YoSHIDA

\section{SUPPORTING INSTITUTIONS}

\author{
UNIVERSITY OF BRITISH COLUMBIA \\ CALIFORNIA INSTITUTE OF TECHNOLOGY \\ UNIVERSITY OF CALIFORNIA \\ MONTANA STATE UNIVERSITY \\ UNIVERSITY OF NEVADA \\ NEW MEXICO STATE UNIVERSITY \\ OREGON STATE UNIVERSITY \\ UNIVERSITY OF OREGON \\ OSAKA UNIVERSITY \\ UNIVERSITY OF SOUTHERN CALIFORNIA
}

\author{
STANFORD UNIVERSITY \\ UNIVERSITY OF TOKYO \\ UNIVERSITY OF UTAH \\ WASHINGTON STATE UNIVERSITY \\ UNIVERSITY OF WASHINGTON \\ $\stackrel{*}{*} \stackrel{*}{*}{ }^{*}{ }^{*}$ \\ CHEVRON RESEARCH CORPORATION \\ TRW SYSTEMS \\ NAVAL WEAPONS CENTER
}

The Supporting Institutions listed above contribute to the cost of publication of this Journal, but they are not owners or publishers and have no responsibility for its content or policies.

Mathematical papers intended for publication in the Pacific Journal of Mathematics should be in typed form or offset-reproduced, double spaced with large margins. Underline Greek letters in red, German in green, and script in blue. The first paragraph or two must be capable of being used separately as a synopsis of the entire paper. It should not contain references to the bibliography. Manuscripts, in duplicate if possible, may be sent to any one of the four editors. Please classify according to the scheme of Math. Rev. 36, 1539-1546. All other communications to the editors should be addressed to the managing editor, Richard Arens, University of California, Los Angeles, California, 90024.

50 reprints are provided free for each article; additional copies may be obtained at cost in multiples of 50 .

The Pacific Journal of Mathematics is published monthly. Effective with Volume 16 the price per volume (3 numbers) is $\$ 8.00$; single issues, $\$ 3.00$. Special price for current issues to individual faculty members of supporting institutions and to individual members of the American Mathematical Society: $\$ 4.00$ per volume; single issues $\$ 1.50$. Back numbers are available.

Subscriptions, orders for back numbers, and changes of address should be sent to Pacific Journal of Mathematics, 103 Highland Boulevard, Berkeley, California, 94708.

PUBLISHED BY PACIFIC JOURNAL OF MATHEMATICS, A NON-PROFIT CORPORATION

Printed at Kokusai Bunken Insatsusha (International Academic Printing Co., Ltd.), 7-17, Fujimi 2-chome, Chiyoda-ku, Tokyo, Japan. 


\section{Pacific Journal of Mathematics \\ Vol. 30, No. $2 \quad$ October, 1969}

Gregory Frank Bachelis, Homomorphisms of annihilator Banach algebras.

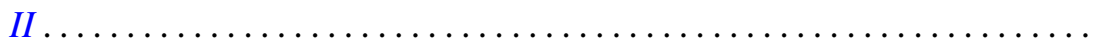

Leon Bernstein and Helmut Hasse, An explicit formula for the units of an algebraic number field of degree $n \geq 2 \ldots \ldots \ldots \ldots \ldots \ldots \ldots . \ldots 29$

David W. Boyd, Best constants in a class of integral inequalities ........ 367

Paul F. Conrad and John Dauns, An embedding theorem for lattice-ordered

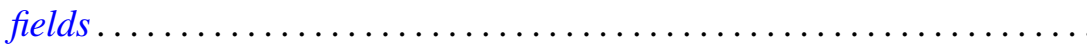

H. P. Dikshit, Summability of Fourier series by triangular matrix

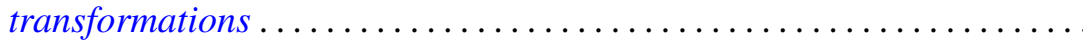

Dragomir Z. Djokovic, Linear transformations of tensor products preserving a fixed rank............................. 411

John J. F. Fournier, Extensions of a Fourier multiplier theorem of Paley . . . 415 Robert Paul Kopp, A subcollection of algebras in a collection of Banach

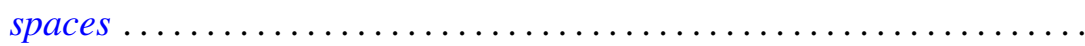

Lawrence Louis Larmore, Twisted cohomology and enumeration of vector bundles ...................................... 437

William Grenfell Leavitt and Yu-Lee Lee, A radical coinciding with the lower radical in associative and alternative rings .................

Samuel Merrill and Nand Lal, Characterization of certain invariant subspaces of $H^{p}$ and $L^{p}$ spaces derived from logmodular

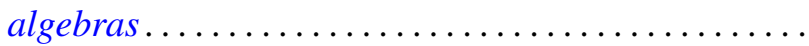

Sam Bernard Nadler, Jr., Multi-valued contraction mappings ....

T. V. Panchapagesan, Semi-groups of scalar type operators in Banach spaces ....................................

J. W. Spellmann, Concerning the infinite differentiability of semigroup motions

H. M. (Hari Mohan) Srivastava, A note on certain dual series equations involving Laguerre polynomials.

Ernest Lester Stitzinger, A nonimbedding theorem of associative algebras................................

J. Jerry Uhl, Jr., Martingales of vector valued set functions ...

John Mays Worrell Jr., On continuous mappings of metacompact $\check{C} e c h$

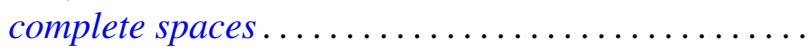

